\title{
Patient Perspectives on the Benefits and Risks of Percutaneous Coronary Interventions: A Qualitative Study
}

This article was published in the following Dove Press journal: Patient Preference and Adherence

\author{
M Pilar Ingle $\mathbb{D}^{\prime}$ \\ William Lammons $\mathbb{D}^{2}$ \\ Rebecca Guigli ${ }^{3}$ \\ Vinay Kini ${ }^{4}$ \\ Daniel D Matlock (D) ${ }^{5}$ \\ Elinor Brereton ${ }^{6}$ \\ Laura D Scherer ${ }^{4}$ \\ 'Graduate School of Social Work, \\ University of Denver, Denver, CO, USA; \\ ${ }^{2}$ Section of Neonatal Medicine, Division \\ of Public Health and Primary Care, \\ Faculty of Medicine, Imperial College \\ London, London, UK; ${ }^{3}$ Data Science to \\ Patient Value, University of Colorado \\ School of Medicine, Aurora, CO, USA; \\ ${ }^{4}$ Division of Cardiology, University of \\ Colorado School of Medicine, Denver, \\ CO, USA; ${ }^{5}$ Division of Geriatric \\ Medicine, University of Colorado School \\ of Medicine, Aurora, CO, USA; ${ }^{6}$ Adult \& \\ Child Consortium for Outcomes \\ Research \& Delivery Science, University \\ of Colorado School of Medicine, Aurora, \\ CO, USA
}

Background: Growing evidence for coronary stents in patients with stable coronary artery disease (CAD) suggests that the benefits of stents are uncertain. The goal of this study was to assess patients' informational needs and how patients react to information about the uncertain benefit of stents to CAD patients.

Methods: Semi-structured qualitative interviews $(\mathrm{N}=20)$ were conducted with patients with stable CAD who received a recent stent. Data were coded and analyzed using a mixed inductive-deductive approach.

Results: Some patients mistakenly believed that the purpose of their stent was to prevent a future heart attack, and few were previously aware of the uncertain benefit. Nearly all patients perceived positive outcomes from their procedure, even if their symptoms persisted. Some patients had difficulty accepting evidence that stents may not reduce the risk of heart attack or reliably improve symptoms. Nonetheless, patients still expressed a desire to receive new information about the uncertain benefits of stents and wanted to have received this information early in their care.

Conclusion: Many patients with stable CAD do not understand the intended benefit of coronary stents and want to be informed of the evidence of uncertain benefit of coronary stents, even if this would not change their decision. Improved communication and patient education tools are needed to better inform patients. An intervention providing patients with this information early has the potential to solve these problems.

Keywords: qualitative research, patient communication, patient education, coronary stents, coronary artery disease

\section{Introduction}

Patients with stable coronary artery disease (CAD) frequently receive percutaneous coronary intervention (PCI, or coronary stents). In the past, it was widely believed that stents reduced the risk of future heart attacks and reduced angina symptoms in patients with stable CAD. However, three recent large randomized controlled trials have called into question the use of stents as a first-line therapy for many patients with stable CAD. The COURAGE trial showed that stents plus optimal medical therapy did not reduce the chance of heart attack or death compared to optimal medical therapy alone. ${ }^{1}$ The ISCHEMIA Trial showed that among patients with moderate or severe ischemia (reduced blood flow to the heart), there were no differences in the rate of death, heart attack, or hospitalization in patients randomized to receive stents versus intensive medical management as initial therapy. ${ }^{2}$
Correspondence: Laura D Scherer Division of Cardiology, University of Colorado School of Medicine, 13199 E. Montview Blvd. Suite 300, Aurora, CO, 80045, USA

Tel + I 303-772-8872

Email Laura.Scherer@cuanschutz.edu
Patient Preference and Adherence 2021:15 721-728 
Finally, the ORBITA trial showed no evidence that stents reduced angina symptoms (measured by exercise time to onset of angina) compared to a sham procedure. ${ }^{3}$ Hence, there is now considerable evidence that stenting provides minimal benefit among selected patients with stable CAD.

The changing state of the evidence for stents for stable angina constitutes an ongoing and sometimes controversial medical reversal in which the expected benefits of stents are changing. This raises the important question of whether and how patients want to learn about the changing evidence. Shared decision making (SDM) and elicitation of patients' preferences for stents may often be appropriate given that the potential for benefit over medical therapy is uncertain. ${ }^{4,5}$ However, talking to patients about the changing evidence and uncertainty around stents is a sensitive issue because management of CAD is a lifelong endeavor. Many patients have never been adequately informed about the benefits of stents, and their prevailing assumption is that the intervention is necessary and life-saving., ${ }^{6,7}$ Moreover, many patients who have received a stent in the past may at some point consider the possibility of another. It may be difficult for patients to hear that stents in many circumstances are optional, likely do not reduce the chance of a heart attack, and may not even reduce symptoms of angina.

There is a paucity of research identifying how to talk to patients about medical reversals in a way that maintains credibility and trust in medicine. In this study, we conducted qualitative interviews of patients who had recently received a coronary stent for stable CAD. Our goal was to take a first step at assessing patients' informational needs and how best to introduce information about the uncertain benefit of stents to CAD patients. We chose this patient population because individuals who have already received a stent, and who were potentially not well informed of the uncertain benefits at the outset, are among those who are most psychologically vulnerable to the negative implications of this medical reversal.

\section{Methods}

We conducted a phenomenological qualitative interview study to explore the perspectives of patients with stable angina who have received an elective stent, with a specific focus on their perceptions of the benefits of their stent and their reactions to the possibility of uncertain benefits suggested by recent trials. The research team- comprised of experienced researchers and physicians in SDM, psychology, and cardiology - provided input at all stages. This study was approved by the University of Colorado Institutional Review Board.

\section{Sample}

Using purposive and convenience sampling, participants were recruited from the cardiology department at a large university medical center. While this sample was not necessarily representative of CAD patients nationwide, this study was not meant to be representative and instead was conceived as a first step toward identifying patients' informational needs and the viability of talking about this medical reversal, in order to inform future national studies and communication interventions. Sampling was conducted until we reached thematic saturation. ${ }^{8}$ Inclusion criteria were patients aged 18-89 with stable coronary artery disease and had received an elective stent in the past 12 months. Patients who were non-English speaking or had a neurological disorder or disease were excluded. Consistent with eligibility criteria from the COURAGE trial, we also excluded patients with acute coronary syndrome, patients who received a stent in the left main or proximal left anterior descending arteries, and patients who received a stent for cardiomyopathy or valvular heart disease. ${ }^{1}$ Hence, all patients in this study were those who received an elective stent with the primary medical goal to address angina symptoms. Eligible participants were identified through their medical records and contacted by phone or email to introduce the study. A total of 46 eligible patients were contacted by phone or email; of those 46, five declined directly, 19 did not respond to recruitment outreach efforts, and 22 agreed to participate. Phone interviews were scheduled and participants were provided with detailed participation and consent information. Due to the low-risk nature of the study and because all recruitment and interviews were conducted over the phone, a waiver of written consent was requested from and approved for this study by the institutional review board. All participants were provided with a consent information sheet prior to their scheduled interview. At the time of the interview, consent information was reviewed indepth with all participants and verbal consent was obtained prior to study commencements. All participants received $\$ 20$ gift cards for their time.

\section{Data Collection}

Research staff extracted clinical data from participants' medical records, including age, left ventricular ejection fraction, Canadian Cardiovascular Society (CCS) Angina 
Score, presence of comorbidities, prior stent placement, and location of the most recent stent. M.P.I., who is an experienced interviewer, conducted all participant interviews between May and September 2019. There were no prior relationships between the interviewer and study participants.

Using a semi-structured interview guide, the first half of the interview focused on patients' understanding of their stent and the information they received from their clinician. The second half of the interview focused on patients' reactions to brief descriptions of the findings from published trials, ${ }^{1,3}$ suggesting uncertain benefit of stents for stable angina with regard to reducing heart attack risk and angina symptoms. Given the potential for this information to evoke negative emotions, we introduced it as a hypothetical. Specifically, participants were told: "There are two potential reasons that one might receive a stent. One is to reduce chance of a heart attack, and one is to reduce chest pain. Suppose there is a major study showing that stents for symptoms like yours did not help prevent heart attacks but only helped lower chest pain symptoms. Then, another major study suggests that stents may not help with chest pain either ...", and then the interview proceeded to questions about how they would react to this evidence, whether they think this information would be important to know about, and whether they think this information would have influenced their decisions. Interviews lasted between 10 and 37 minutes. All interviews were conducted over the phone. Basic demographic information-including race, ethnicity, gender, employment status, and income level-were also collected. All interviews were audio recorded and transcribed verbatim. Investigator triangulation was used throughout the data collection process by means of interview debriefings with L.S. and V.K. in attempts to reduce bias and broaden understanding of emerging themes from the interviews. ${ }^{9,10}$

\section{Analysis}

While this study is phenomenological in nature, given the specific context from which we explored these partipicants' experiences analyses were conducted using an iterative mixed inductive-deductive approach. Content analysis was performed to establish themes from the data. ${ }^{11}$ All coding and analyses were conducted by the primary analytic team (M.P.I., W.L., and L.S.). In the first stage, the research team developed an initial codebook using a priori concepts directly from the interview guide and interviews were coded using a structural coding process. ${ }^{12}$ Domains analyzed in this stage included experiences of symptoms prior to a stent placement, the process of receiving or seeking information about the procedure, involvement in decision making, and responses to information about evidence for uncertain benefits of a stent.

In the second stage, the team used a generalized inductive process of open coding to provide conceptual descriptions of patient experiences with getting a stent and their reactions to uncertain benefits. ${ }^{13}$ The analytic team met regularly to discuss any discrepancies, emerging themes, and to refine and finalize the codebook. Using the final codebook, two members of the team (W.L. and M.P.I.) independently coded five interviews that were selected to represent participants across the span of the study from the sample to ensure coding agreement with consultation from the rest of the team as needed. Once coding agreement was achieved, all interviews were coded using Dedoose qualitative software (https://www.dedoose.com) and themes were extracted.

\section{Results}

Twenty-two participants agreed to participate in the study, and a total of 20 participants were interviewed. One participant dropped out prior to the interview, and another was dropped from the study because the patient had a left ventricular assist device and different clinical criteria for receipt of PCI as a result. Participant demographics are displayed in Table 1. Participants ranged between the ages of 39 and 88 , and the majority were white (60\%), male (85\%), and college educated (80\%).

Four main themes emerged from the analysis of the interviews: inconsistent understanding of the stent's purpose, positive perceptions and mixed outcomes, reactions to information about uncertain benefits, and importance of being informed. These themes are further elaborated in conjunction with participant quotations below.

\section{Inconsistent Understanding of the Stent's Purpose}

All of these patients' medical records suggested that the primary purpose for receiving a stent in their case was symptom relief. Nonetheless, patients described a variety of purposes they believed their stent served, including clearing blockages, preventing heart attacks, extending life, and reducing angina symptoms. A few patients explicitly articulated a belief that a stent would reduce their chance of a heart attack: 
Table I Participant Demographics and Clinical Characteristics $(n=20)$

\begin{tabular}{|c|c|c|}
\hline & $\mathbf{n}$ & Mean/Percentage \\
\hline Age (39-88) & 20 & $64(S D=10.5)$ \\
\hline Sex (male) & 17 & $85 \%$ \\
\hline Married & 14 & $70 \%$ \\
\hline \multicolumn{3}{|l|}{ Race/Ethnicity } \\
\hline White & 12 & $60 \%$ \\
\hline American Indian or Alaskan Native & 4 & $20 \%$ \\
\hline Asian or Pacific Islander & 1 & $5 \%$ \\
\hline Black or African American & 1 & $5 \%$ \\
\hline Hispanic/Latinx & 3 & $15 \%$ \\
\hline Another race or multiracial & 2 & $10 \%$ \\
\hline \multicolumn{3}{|l|}{ Education } \\
\hline High school graduate or GED & 2 & $10 \%$ \\
\hline Some college & 2 & $10 \%$ \\
\hline College graduate or post-graduate & 16 & $80 \%$ \\
\hline \multicolumn{3}{|l|}{ Employment Status } \\
\hline Full or part time & 4 & $20 \%$ \\
\hline Retired & 14 & $70 \%$ \\
\hline Student or other & 2 & $10 \%$ \\
\hline \multicolumn{3}{|l|}{ Income } \\
\hline$\geq \$ 60,000$ & 6 & $30 \%$ \\
\hline$<\$ 60,000$ & 14 & $70 \%$ \\
\hline Diabetes & 4 & $20 \%$ \\
\hline Hypertension & 10 & $50 \%$ \\
\hline Hyperlipidemia & 8 & $40 \%$ \\
\hline Current smoker & 0 & $0 \%$ \\
\hline Prior myocardial infarction & 7 & $35 \%$ \\
\hline Prior stent & 9 & $45 \%$ \\
\hline \multicolumn{3}{|l|}{ Left ventricular ejection fraction } \\
\hline Normal & 15 & $75 \%$ \\
\hline Mildly reduced & 2 & $10 \%$ \\
\hline Moderately reduced & 2 & $10 \%$ \\
\hline Severely reduced & 1 & $5 \%$ \\
\hline \multicolumn{3}{|l|}{ Angina CCS class } \\
\hline Class I & 4 & $20 \%$ \\
\hline Class II & 7 & $35 \%$ \\
\hline Class III & 9 & $45 \%$ \\
\hline \multicolumn{3}{|l|}{ Stented artery } \\
\hline LAD & 14 & $70 \%$ \\
\hline LCx & 1 & $5 \%$ \\
\hline RCA & 5 & $25 \%$ \\
\hline \multicolumn{3}{|l|}{ Stented artery location } \\
\hline Proximal & 6 & $30 \%$ \\
\hline Mid & 11 & $55 \%$ \\
\hline Distal & 3 & $15 \%$ \\
\hline
\end{tabular}

Abbreviations: GED, General Education Development; CCS, Canadian Cardiovascular Society; LAD, left anterior descending; LCx, left circumflex; RCA, right coronary artery.
"Yeah, like imminent heart attack because ... there was some kind of blockage that they couldn't see." (Male, Age 62)

"Um, to, you know, prevent me from having a heart attack due to the plaque buildup." (Male, Age 54)

Several patients stated correctly that, per their understanding, that a stent would clear the plaque buildup blocking blood flow from their arteries to the heart. However, these patients were sometimes vague about what they believed was the specific benefit of unblocking their arteries. Other patients appeared to have a good understanding of using a stent for symptom control:

"Uh, getting rid of my chest pains. That's the ultimate goal." (Male, Age 63, a)

"That my artery would be unblocked, my blood flow would be better, I would feel better and stronger." (Female, Age 70)

In some cases, patients reported that their understanding of symptoms were clarified during discussions with clinicians. However, several patients reported that their clinician did not explain the purpose of a stent at all, or described it in brief detail.

Well ... it's important that, uh, people know more about what's gonna happen. Uh, if I have one criticism, it was I really was never told the full, complete story before everything happened. You know? You don't have all your questions answered. (Male, Age 88)

\section{Positive Perceptions and Mixed Outcomes}

Nearly every patient had a positive perception of their stent results, including the patients who reported not experiencing angina symptoms to begin with (and who therefore could not have benefitted from the stent). Some patients reported having reduced chest pain, increased energy, and greater ease with breathing. Yet, some patients who reported improvements also reported mixed outcomes including continued experiences of symptoms. One participant reported actually felt worse following her procedure, noting that her symptoms were more frequent after receiving the stent.

Because I still had chest pains, except the problem was that I was having chest pains without the exertion. Before I would only have chest pains with the exertion and then after the procedure they came on more without exertion. (Female, Age 67) 
Some patients also highlighted a long recovery process following the stent procedure, particularly due to the bruising and shortness of breath associated with blood thinner medications.

\section{Reactions to Information About Uncertain Benefits}

Few participants were aware of the uncertain benefits of stents prior to their participation in the study. Participants' immediate reactions to the evidence generally fell into three categories: deferring to their doctor's knowledge, disbelief, and concern. For example, some participants reacted to the evidence by emphasizing their trust in their doctor, believing they have the best information and have the patient's best interest in mind.

I basically would have just gone with what the doctor said. You know, they know a lot more than I do, so I just basically went with what they thought was best for me ... No, I don't think I'd change anything, I'd just keep on doing what I've been doing. (Male, Age 66)

Others dismissed the evidence, deferring to their own experience of feeling better after the procedure.

"I just know I feel better, so that's all I can say, you know? So, I'm not bothered by those studies." (Male, Age 53)

Some participants even expressed disbelief or postulated the possibilities that there may be bias or clinical error in the trials.

Um, well, I would definitely read them ... and how would they make me feel? ... Um, I'd be stunned, knowing what I know. I'd be stunned and looked, look for, uh, clinical errors in the data ... it would be disbelief. Yeah, it would be disbelief. (Male, Age 63, b)

"You can find- as I'm sure you know, opposing side of any position that anybody takes on anything. You can always find somebody with a differing view." (Male, Age 72)

While many respondents questioned or dismissed the information, other participants responded with disappointment or concern.

"I suppose it would be a little disappointing. You know, I guess it would make one question a little bit uh, is it worth having it." (Male, Age 64, a)

\section{The Importance of Being Informed}

When presented with information about uncertain benefits, the majority of participants expressed that this information would be important to know prior to receiving the stent. At the same time, most participants stated that the information from these studies would not have impacted their decisions. Many stated that they would defer to their physician's recommendation, but would still like to have all of the information available:

I want to know everything that's possible, everything that could go wrong, everything that they're going to do and as much information as they can share with me and don't candy coat it. Be honest with me. (Male, Age 71)

"I think it's highly important. I mean, you should know what's going on before you even get stuff like that done." (Male, Age 66)

When asked at what point information about uncertain benefits should be presented in the decision making process, participants gave varying answers, from the first onset of symptoms to right before the stent procedure. However, most participants expressed that the information should be discussed as early as possible. The few participants who stated that they would not want to receive the information provided by the studies said that the amount of detail would be overwhelming or unnecessary, and that their physician's recommendation was the most important.

"Um, I don't want to know all the information. I don't need to go into the weeds." (Male, Age 46).

Most patients described their own participation in the decision-making process, though some patients experienced the process as being driven by clinicians. Several patients explicitly deferred decisions surrounding the stent procedure to clinicians.

"I think [the doctor] said, 'You're getting one.' I don't remember them ever explaining what it was. I still don't even know what it is." (Male, Age 39)

"Uh, talked about it, asked if it sounded like a good idea, and I agreed with [the doctor]. You know, he's the expert." (Male, Age 63, a)

\section{Discussion}

In this qualitative study the majority of patients who had received a stent for stable coronary artery disease were unaware of uncertain benefits of stents as illustrated by the results of the COURAGE and ORBITA trials (the ISCHEMIA trial was published in the months after these interviews were conducted) ${ }^{1,3}$ Only a couple of participants in this study were previously aware of the evidence of uncertain benefits, particularly the fact that a stent probably does not reduce the risk of future heart attack or angina symptoms (a finding which is consistent with past patient surveys ${ }^{6,7}$ ). Patients 
generally believed that the stent had benefited them, even if they experienced continued angina symptoms after the stent or if they did not have angina symptoms before the stent. ${ }^{14}$

The most important contribution of this study is how patients reacted to evidence that stents do not reduce the risk of heart attack or angina symptoms. Many patients responded by expressing trust in their doctor's judgment. However, some patients expressed disbelief and concern. Disbelief, in particular, could present a communication challenge. Disbelief can be a defensive coping mechanism to help individuals grapple with unwelcome information. ${ }^{15,16}$ People also express disbelief when information is inconsistent with their personal experience, ${ }^{17}$ and in this case, many patients reported a subjective experience of benefit from their stent. Disbelief may be more commonly expressed by patients who have already received a stent, which suggests the importance of informing patients early in their care trajectory and prior to any procedures, when possible.

The information about uncertain benefits was presented as a hypothetical in all interviews; however, if asked directly by the participants whether the information was real, we confirmed the information was based on real trials. There did not appear to be any noticeable differences among reactions to the information between the few participants who asked about the validity of this information versus the participants who did not.

A few patients were noticeably distressed by the information. While we did not probe deeply into the exact cause of their distress, in at least one case it appeared to be the result of a renewed feeling of susceptibility to a future heart attack. On one hand, this kind of revelation might help patients to appreciate the importance of medications and lifestyle changes for reducing heart attack risk. On the other hand, this finding suggests a need to support patients' anxiety about heart attack risk when conveying evidence about uncertain benefits of stents, especially among patients who have already received a stent (but also perhaps among patients who are receiving their first stent).

Critically, most patients thought that it was important that patients receive information about the evidence prior to receiving a stent. When asked when they would hypothetically like to receive such information, the resounding answer was the earlier the better, even if it would not change the care that they ultimately received. This suggests the need for patient education and inviting patients to be more involved in the decision-making process.

Patient-centered care is defined as care that is "respectful of and responsive to individual patient preferences, needs, and values, and ensuring that patient values guide all clinical decisions". ${ }^{18}$ The decisions described by these patients were generally not patient-centered, insofar as patients were minimally involved in the decision and not well informed of the potential benefits and harms. While decision aids have been developed to educate patients about stents and promote patient-centeredness at the time of a stent decision, ${ }^{19}$ these DAs have not been widely implemented. The present study suggests that patients want to receive, and could potentially benefit from receiving, information about stents much earlier in their care trajectory. For example, information about stents could be delivered in the context of a new diagnosis, with the educational goal being to provide patients with information about their diagnosis and what to expect in terms of different treatments that are likely to be offered, both immediately and in the years to come. Altogether, these results point to patients' desire for information, and an ethical responsibility to deliver this information to patients even if it does not influence their decisions. Moreover, delivering this information earlier means providing it through a pamphlet or discussion and not merely as part of an informed consent document just prior to a stent procedure.

\section{Study Limitations}

The study sample was limited to a single medical center and was predominantly white, male and educated. A less educated sample might be even less informed about the purpose and expected benefits of stents. Additionally, a sample with a greater representation of marginalized racial and ethnic identities might experience more distrust and alienation if it is revealed that the purpose of the stent was quite different from what they were expecting. ${ }^{20}$

The present study should be interpreted as a starting point for understanding the implications of communicating this medical reserval. Future research should explore the issue of communicating about this medical reversal using more representative samples of patients, potentially with quantitative or mixed methods. Moreover, all interview participants imagined how the new evidence would have changed their decisions, which may reflect hesitance to engage deeply with an idea that might cause regret about their past decision to get a stent. Therefore, informational 
interventions may have a larger effect on patients' decisions than what these interviews suggest.

\section{Conclusions}

Our study supports previous findings of patients' misunderstanding of the purpose of receiving a stent and expectations that the procedure will prevent future heart attacks. Most patients in our sample expressed a desire to receive information about the most current evidence, including information about uncertain benefits of stents, early on in their care trajectory in order to make an informed decision about their procedure (even if this information would not ultimately change their decision).

An intervention educating patients on the risks and benefits of stents may improve awareness of the impact of these decisions on their future health and well-being and increase patient activation. The time at which patients stated they would prefer this information varies, but the overall consensus is that sooner is better.

\section{Abbreviations}

CAD, coronary artery disease; PCI, percutaneous coronary intervention; CCS, Canadian Cardiovascular Society.

\section{Ethics Statement}

This study was approved by the University of Colorado Institutional Review Board (COMIRB\# 19-0012) and conducted in accordance with the Declaration of Helsinki. A waiver of written consent was approved for the study, and all participants received a consent information form and gave verbal consent to participate prior to study commencement.

\section{Consent for Publication}

Patient consent included publication of anonymized responses. All patient/personal identifiers have been removed or disguised so the patient/person(s) described are not identifiable and cannot be identified through the details of the story.

\section{Acknowledgments}

We could not have done this work without the participants willing to share their stories.

\section{Author Contributions}

All authors made a significant contribution to the work reported, whether that is in the conception, study design, execution, acquisition of data, analysis and interpretation, or in all these areas; took part in drafting, revising or critically reviewing the article; gave final approval of the version to be published; have agreed on the journal to which the article has been submitted; and agree to be accountable for all aspects of the work.

\section{Funding}

The study was funded by start up funds allocated to Dr. Scherer by the University of Colorado. This project was supported by the Data Science to Patient Value (D2V) initiative funded by the University of Colorado School of Medicine Dean's Transformational Research Funding. Dr. Kini is supported by an NIH Career Development Award (K23HL143208) and a Greenwall Foundation award, both unrelated to the content of this study.

\section{Disclosure}

The authors declare that they have no competing interests.

\section{References}

1. Boden WE, O'Rourke RA, Teo KK, et al. The evolving pattern of symptomatic coronary artery disease in the United States and Canada: baseline characteristics of the Clinical Outcomes Utilizing Revascularization and Aggressive DruG Evaluation (COURAGE) trial. Am J Cardiol. 2007;99(2):208-212. doi:10.1016/j.amjcard.2 006.07 .082

2. Maron DJ, Hochman JS, Reynolds HR, et al. Initial invasive or conservative strategy for stable coronary disease. $N$ Engl J Med. 2020;382(15):1395-1407. doi:10.1056/NEJMoa1915922

3. Al-Lamee R, Thompson D, Dehbi H-M, et al. Percutaneous coronary intervention in stable angina (ORBITA): a double-blind, randomised controlled trial. Lancet. 2018;391(10115):31-40. doi:10.1016/S01406736(17)32714-9

4. Chhatriwalla AK, Decker C, Gialde E, et al. Developing and testing a personalized, evidence-based, shared decision-making tool for stent selection in percutaneous coronary intervention using a pre-post study design. Circ Cardiovasc Qual Outcomes. 2019;12(2):e005139. doi:10.1161/CIRCOUTCOMES.118.005139

5. Coylewright M, Shepel K, LeBlanc A, et al. Shared decision making in patients with stable coronary artery disease: PCI choice. PLoS One. 2012;7(11):e49827. doi:10.1371/journal.pone.0049827

6. Goff SL, Mazor KM, Ting HH, Kleppel R, Rothberg MB. How cardiologists present the benefits of percutaneous coronary interventions to patients with stable angina: a qualitative analysis. JAMA Intern Med. 2014;174(10):1614-1621. doi:10.1001/jamainter nmed.2014.3328

7. Rothberg MB, Sivalingam SK, Kleppel R, Schweiger M, Hu B, Sepucha KR. Informed decision making for percutaneous coronary intervention for stable coronary disease. JAMA Intern Med. 2015;175 (7):1199-1206. doi:10.1001/jamainternmed.2015.1657

8. Moser A, Korstjens I. Series: practical guidance to qualitative research. Part 3: sampling, data collection and analysis. Eur J Gen Pract. 2018;24(1):9-18. doi:10.1080/13814788.2017.1375091

9. Denzin NK. Sociological Methods: A Sourcebook. Routledge; 2017.

10. Carter N, Bryant-Lukosius D, DiCenso A, Blythe J, Neville AJ. The use of triangulation in qualitative research. Oncol Nurs Forum. 2014;41(5):545-547. doi:10.1188/14.ONF.545-547

11. Krippendorff K. Content Analysis: An Introduction to Its Methodology. Sage publications; 2018. 
12. Saldaña J. The Coding Manual for Qualitative Researchers. Sage; 2016.

13. Thomas DR. A general inductive approach for analyzing qualitative evaluation data. Am J Eval. 2006;27(2):237-246. doi:10.1177/ 1098214005283748

14. Saxon JT, Chan PS, Tran AT, et al. Comparison of patient-reported vs physician-estimated angina in patients undergoing elective and urgent percutaneous coronary intervention. JAMA Netw Open. 2020;3(6): e207406. doi:10.1001/jamanetworkopen.2020.7406

15. McQueen A, Vernon SW, Swank PR. Construct definition and scale development for defensive information processing: an application to colorectal cancer screening. Health Psychol. 2013;32(2):190. doi: $10.1037 / \mathrm{a} 0027311$

16. Kunda Z. The case for motivated reasoning. Psychol Bull. 1990;108 (3):480. doi:10.1037/0033-2909.108.3.480
17. Druckman JN, McGrath MC. The evidence for motivated reasoning in climate change preference formation. Nat Clim Change. 2019;9 (2):111-119. doi:10.1038/s41558-018-0360-1

18. Baker A. Crossing the Quality Chasm: A New Health System for the 21st Century. Vol. 323. British Medical Journal Publishing Group; 2001.

19. Coylewright M, Dick S, Zmolek B, et al. PCI choice decision aid for stable coronary artery disease: a randomized trial. Circ Cardiovasc Qual Outcomes. 2016;9(6):767-776. doi:10.1161/CIRCOUT COMES.116.002641

20. Armstrong K, Putt M, Halbert $\mathrm{CH}$, et al. Prior experiences of racial discrimination and racial differences in health care system distrust. Med Care. 2013;51(2):144. doi:10.1097/MLR.0b013e318273 $10 \mathrm{a} 1$

\section{Publish your work in this journal}

Patient Preference and Adherence is an international, peer-reviewed, open access journal that focusing on the growing importance of patient preference and adherence throughout the therapeutic continuum. Patient satisfaction, acceptability, quality of life, compliance, persistence and their role in developing new therapeutic modalities and compounds to optimize clinical outcomes for existing disease states are major areas of interest for the journal. This journal has been accepted for indexing on PubMed Central. The manuscript management system is completely online and includes a very quick and fair peer-review system, which is all easy to use. Visit http:// www.dovepress.com/testimonials.php to read real quotes from published authors. 